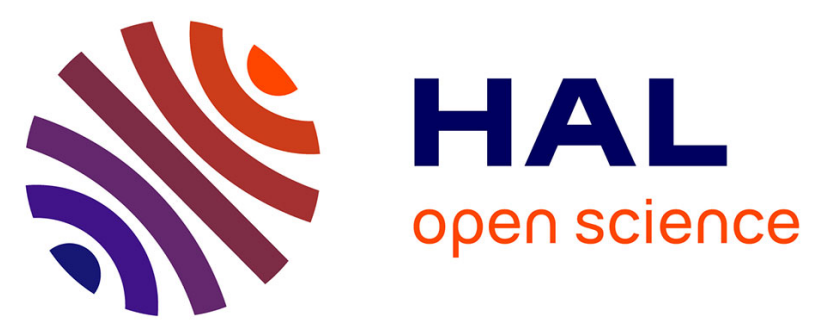

\title{
Evaluation of target non-uniformity and dispersion effects on energy measurement resolution in NUMEN experiment
}

Federico Pinna, Daniela Calvo, Matteo Campostrini, Vittoria Capirossi, Franck Delaunay, Maria Fisichella, Felice Iazzi, Valentino Rigato

\section{To cite this version:}

Federico Pinna, Daniela Calvo, Matteo Campostrini, Vittoria Capirossi, Franck Delaunay, et al.. Evaluation of target non-uniformity and dispersion effects on energy measurement resolution in NUMEN experiment. Phys.Scripta, 2020, 95 (9), pp.094002. 10.1088/1402-4896/aba779 . hal-02917252

\section{HAL Id: hal-02917252 \\ https://hal.science/hal-02917252}

Submitted on 11 Oct 2021

HAL is a multi-disciplinary open access archive for the deposit and dissemination of scientific research documents, whether they are published or not. The documents may come from teaching and research institutions in France or abroad, or from public or private research centers.
L'archive ouverte pluridisciplinaire HAL, est destinée au dépôt et à la diffusion de documents scientifiques de niveau recherche, publiés ou non, émanant des établissements d'enseignement et de recherche français ou étrangers, des laboratoires publics ou privés. 


\title{
PAPER
}

Evaluation of target non-uniformity and dispersion

Recent citations effects on energy measurement resolution in NUMEN experiment

\author{
The NUMEN Project: An Update of the \\ Facility Toward the Future Experimental \\ Campaigns \\ Francesco Cappuzzello et al
}

To cite this article: Federico Pinna et al 2020 Phys. Scr. 95094002

View the article online for updates and enhancements. 


\title{
Evaluation of target non-uniformity and dispersion effects on energy measurement resolution in NUMEN experiment
}

\author{
Federico Pinna ${ }^{1,2}$, Daniela Calvo ${ }^{2}$, Matteo Campostrini ${ }^{3}$, \\ Vittoria Capirossi ${ }^{1,2}$, Franck Delaunay ${ }^{4}$, Maria Fisichella ${ }^{2}$, Felice lazzi ${ }^{1,2}$ and \\ Valentino Rigato ${ }^{3}$ \\ ${ }^{1}$ Politecnico di Torino, Torino, Italy \\ ${ }^{2}$ INFN-Sezione di Torino, Torino, Italy \\ ${ }^{3}$ INFN_LNL, Legnaro, Padova, Italy \\ ${ }^{4}$ LPC Caen, Normandie Université, ENSICAEN, UNICAEN, CNRS/IN2P3, Caen, France \\ E-mail: federico.pinna@polito.it and federico.pinna@to.infn.it
}

Received 16 April 2020, revised 29 June 2020

Accepted for publication 20 July 2020

Published 31 July 2020

\begin{abstract}
In the NUMEN Experiment, Double Charge Exchange (DCE) reactions will be studied to get very precise measurements of their cross sections and final state levels. The interest for these reactions lies in the possibility for some nuclides to have DCE with initial and final states identical to those of the Neutrinoless Double $\beta$-Decay. To reach a good precision in the energy measurements, high statistics is needed and severe constraints about the target thickness must be satisfied. A $50 \mu \mathrm{A}$ intense ion beam will provide the desired statistics, while posing the problem of dissipating the massive heat generated in the target. It is therefore necessary to design a suitable cooling system, which must affect the particles' energy as little as possible. Said energy is already influenced by the current setup. The Superconducting Cyclotron (SC) and the MAGNEX Spectrometer introduce an error on the particles' energy by $1 / 1000$ th (FWHM value) of its average energy. In the target, the main sources of error are straggling of projectiles and reaction products, and the dispersion effect. Both closely depend on the target thickness, which must be of the order of few hundred nanometres. In addition, the two effects are worsened if the target thickness is not uniform. The solution to these problems has been found by backing the target isotope with relatively thin substrate of Highly Oriented Pyrolytic Graphite (HOPG). Its thermodynamic properties fit the cooling requirements and can be as thin as $450 \mu \mathrm{g} \mathrm{cm}^{-2}$. The further straggling suffered by the ejectiles is tolerable, falling within the resolution requirements. Samples are deposited by using Electron Beam Evaporation: results obtained for Sn and Te are checked by Scanning Electron Microscopy (SEM). A quantitative evaluation of the samples' thickness has been performed by Alpha-Particle Transmission (APT) and Rutherford Backscattering Spectrometry (RBS) measurements. A Monte Carlo code has been implemented to estimate the ejectiles energy distribution using the experimental measurements as input. Results from characterization and simulations help in optimizing the target thickness and the energy resolution of reaction products.
\end{abstract}

Keywords: double charge exchange, Neutrino-less double $\beta$-Decay, target thickness uniformity, isotope deposition, scanning electron microscope, rutherford backscattering spectrometry, alphatransmission measurements

(Some figures may appear in colour only in the online journal) 


\section{Introduction}

The study of rare nuclear reactions requires a sufficiently good precision in the measurement of the physical quantity of interest, e.g. energy of particles. This is the case of the NUMEN experiment, in which the energy of the reaction products, together with other quantities, must be measured with high precision [1]. The main goal of NUMEN is to obtain experimental information about the Nuclear Matrix Elements (NME) of heavy-ion induced Double Charge Exchange reactions [2], whose initial and final states are the same of the corresponding Neutrino-less Double Beta decays $(0 \nu \beta \beta)$, i.e.:

$$
\begin{aligned}
& \text { DCE reaction } \\
& \left(A_{1}, Z_{1}\right)+\left(A_{2}, Z_{2}\right) \rightarrow\left(A_{1}, Z_{1}+2\right)+\left(A_{2}, Z_{2}-2\right) \\
& 0 \nu \beta \beta\left(\beta^{+} \text {decay }\right) \\
& \left(A_{2}, Z_{2}\right) \rightarrow\left(A_{2}, Z_{2}-2\right)+2 \beta^{+} \\
& D C E \text { reaction } \\
& \left(A_{1}, Z_{1}\right)+\left(A_{2}, Z_{2}\right) \rightarrow\left(A_{1}, Z_{1}-2\right)+\left(A_{2}, Z_{2}+2\right) \\
& 0 \nu \beta \beta\left(\beta^{-} \text {decay }\right) \\
& \left(A_{2}, Z_{2}\right) \rightarrow\left(A_{2}, Z_{2}+2\right)+2 \beta^{-}
\end{aligned}
$$

In reactions (1) and (3) the target nucleus, labelled by index 2 , transforms, by DCE with projectile, into the same final nucleus of the Double $\beta$-Decay without any accompanying neutrino shown in formulas (2) and (4). Projectiles used in NUMEN are ${ }^{18} \mathrm{O}$ and ${ }^{20} \mathrm{Ne}$ ion beams, whose energy ranges from 15 to $60 \mathrm{MeV} / \mathrm{A}$. Two preliminary sets of targets, ${ }^{116} \mathrm{Sn},{ }^{76} \mathrm{Se}$ isotopes for ${ }^{18} \mathrm{O}$ beam and ${ }^{116} \mathrm{Cd},{ }^{76} \mathrm{Ge},{ }^{130} \mathrm{Te}$ isotopes for ${ }^{20} \mathrm{Ne}$, are foreseen in the first phases of the experiment. The full list of the foreseen targets is reported in [2].

The required resolution in the energy measurement of the ejectiles depends on the target isotope, but in most cases it is close to $0.5 \mathrm{MeV}$. Considering the error introduced by the experimental setup (Superconducting Cyclotron and track reconstruction in MAGNEX spectrometer, each one introducing an error equal to $1 / 1000$ of the beam energy in FWHM), not much room is left for the error due to the target. Such an error depends on the target material, of course, but also on the thickness: the thicker the target, the worse is the energy resolution, as will be described in more detail in the following sections. Therefore, it is mandatory to produce thin targets. On the other hand, the searched DCE cross sections are about only few $\mathrm{nb} / \mathrm{sr}$ up to few $\mu \mathrm{b} / \mathrm{sr}$, and a large number of reactions are needed to get statistically significant data. Given the very small target thickness, the only viable way is to use very intense ion beams. The first downside of this approach is the massive generation of heat in the target, which by no means can endure such a thermal stress [3].

The issue has been solved by using a special graphite backing, made of Highly Oriented Pyrolytic Graphite (HOPG), which has a very high in-plane thermal conductivity $(1900 \pm 100 \mathrm{~W} / \mathrm{m} \cdot \mathrm{K}$ at room temperature). Aside from thermal management purposes, the graphite backing will also work as mechanical support for the target and as post-stripper for the reaction products. This kind of graphite can be as thin as $450 \mu \mathrm{g} \mathrm{cm}^{-2}$. Unfortunately, adding a further layer after the target affects the ejectile energy and consequently the energy resolution, mainly for the straggling inside the graphite.

In the following sections, the techniques used to characterize the targets will be described. The effects influencing the energy resolution due to the target and HOPG thickness will be discussed in section 4, together with the effects coming from other sources.

Results from characterization of target prototypes will be shown. These results are used in a Monte Carlo code to estimate the energy distribution of reaction products. Simulations outcome for $\mathrm{Sn}$ and Te prototypes will be reported.

\section{Target and Substrate influence on energy resolution}

As mentioned above, the final energy of each ion is affected by statistical effects due to the experimental setup and to the target system (target and HOPG substrate). In the facility upgrade, a great effort is being dedicated in keeping the effects of the equipment close to the current value $(1 / 1000$ of the beam energy in FWHM). However, the loss of resolution due to the equipment cannot be mitigated past a certain point. Among all, the target assembly (target and HOPG substrate) is the source of uncertainty that can be reduced at most. Here, straggling and dispersion are the two main factors affecting the projectile energy. Dispersion is due to the impossibility of knowing where the reaction happens inside the target, while straggling is due to the statistical fluctuation around the average energy loss. Both effects become more relevant with increasing the thickness of the crossed mediums; also, they are worsened if the target and HOPG thickness is not uniform. Given these premises, the optimization of the target system physical characteristics is of paramount importance to have the best trade off among cooling, target thickness and energy resolution.

Therefore, a thorough characterization procedure is mandatory to correlate deposition parameters and samples characteristics. The techniques used are FESEM, Alpha-Particle Transmission (APT) and Rutherford Backscattering Spectrometry (RBS). Atomic Force Microscopy (AFM) was also considered to further investigate the samples' surface, but it proved to be unsuited for the research purposes and was soon abandoned.

\section{Target characterization}

Samples showed in this work are elemental Te and Sn film deposited by electron beam evaporation on HOPG substrates. The thicker graphite (nominal thickness $2200 \mu \mathrm{g} \mathrm{cm}^{-2}$ ) was used at the beginning of the work to test the HOPG as a substrate; meanwhile, a thinner HOPG type, $1100 \mu \mathrm{g} \mathrm{cm}^{-2}$ thick, was found to be commercially available, so the initial results were then transferred on the thinner HOPG. Tellurium samples presented here are called A14 (on $2200 \mu \mathrm{g} \mathrm{cm}^{-2}$ 


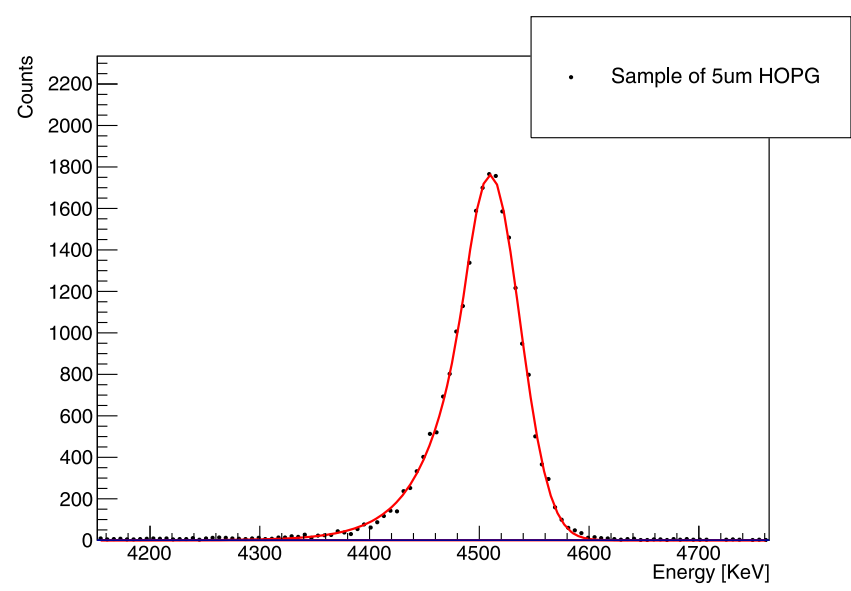

Figure 1. A standard APT data set of a $1100 \mu \mathrm{g} \mathrm{cm}^{-2}$ (nominal thickness) thick HOPG sample. The collected data are shown in black dots, while the red solid line is a Crystal-Ball fit [5].

HOPG) and B10 (on $1100 \mu \mathrm{g} \mathrm{cm}^{-2} \mathrm{HOPG}$ ); tin samples are named A20 (on $2200 \mu \mathrm{g} \mathrm{cm}^{-2}$ HOPG) and B13 (on $\left.1100 \mu \mathrm{g} \mathrm{cm}^{-2} \mathrm{HOPG}\right)$. The target morphology is influenced by several factors, such as the deposition parameters and conditions, the material properties, the substrate surface's properties. All of them must be considered or tuned to obtain a sufficiently uniform deposition and reduce the target contribution to the energy resolution.

As first step, the HOPG foil is characterized by APT technique measurements. Alpha-Particle Transmission is a non-destructive characterization technique that allows to get precise quantitative information on the sample thickness and its thickness uniformity. In this characterization procedure, a collimated ion beam of known energy crosses the sample, losing energy in the process. A Si detector, placed downstream the sample, is used to measure the residual energy of the ions, thanks to which the thickness distribution of the sample can be evaluated. The ion beam, made of alpha particles, is provided by a radioactive ${ }^{241} \mathrm{Am}$ source. The detailed characterization procedure is described in [4]. From such measurements, the HOPG's average thickness and disuniformity are evaluated. In particular, the average thickness is taken as the most probable value in the data distribution, while the disuniformity is evaluated through the distribution's standard deviation. A typical APT plot of a HOPG substrate is shown in figure 1.

The analysis of several HOPG samples, with nominal thickness of $1100 \mu \mathrm{g} \mathrm{cm}^{-2}$, suggests that a certain degree of roughness is intrinsic in the graphite substrates. The measured average thickness is $1208 \mu \mathrm{g} \mathrm{cm}^{-2}$, with an average disuniformity of $2 \%$ of the total thickness. The low-energy tail is more pronounced than the high-energy one; this can be due to the combined effect of large bumps on the HOPG surface and the higher energy loss of the $\alpha$ particle at lower energy. Details on the roughness estimation are reported in [4].

After the backing has been characterized, the thin film target is deposited and qualitatively characterized by Electron Microscopy, to quickly check the complete substrate coverage and have a first insight on the film structure.
SEM images of the Te samples, reported in figure 2, show that small structures some tens of $\mathrm{nm}$ in size cover an overall flat and compact background. Sample B10 (figure 2(a)) shows no particular differences from sample A14 (figure 2(b)). On the contrary, the $\mathrm{Sn}$ films appear more uneven. In sample A20, reported in figure 3(a), the substrate is completely covered, but the background is topped by relatively large structures. On the other hand, sample B13 appears overall flat, but deep trenches some tens of nm large can be seen (figure 3(b)). For comparison, the SEM image of a graphite substrate is also reported (figure 4). Similarly to the HOPG case, the quantitative characterization of samples thickness and thickness unevenness is performed by using APT. Data collected before and after the deposition of the samples are shown in figures 5 and 6, for Te and Sn samples respectively. Using previously performed measurements of the substrate, the HOPG contribution can be subtracted to indirectly obtain the target average thickness and disuniformity. The plot reports data collected before (black dots) and after (orange dots) the deposition of the target. The data are fitted using a Crystal-ball function, a function developed for energy loss processes. It is composed by a Gaussian part, which fits the peak of the distribution, and a power law, for the low energy tail [5]. Crystal Ball fits (red solid line) are well superimposed to the data; since the core of the function is a Gaussian, it is fair to consider the sample disuniformity to follow a Gaussian distribution too. A formal explanation will be provided in an incoming work.

The thickness of samples A20 and B13 resulted to be $160.6 \mu \mathrm{g} \mathrm{cm}^{-2}$ and $131 \mu \mathrm{g} \mathrm{cm}^{-2}$, respectively. The thickness distribution's standard deviation has been estimated to be equal to $70.8 \mu \mathrm{g} \mathrm{cm}^{-2}$ for A20 and $85.4 \mu \mathrm{g} \mathrm{cm}^{-2}$ for B13 (about $44 \%$ and $65 \%$ of the average thicknesses). For both the Te samples, the measured thickness was $268 \mu \mathrm{g} \mathrm{cm}^{-2}$, sample A14 having a standard deviation of $16 \mu \mathrm{g} \mathrm{cm}^{-2}$ and sample B10 having a standard deviation of $29.5 \mu \mathrm{g} \mathrm{cm}^{-2}$ (about $6 \%$ and $11 \%$ of the respective average thicknesses). The spectra reported in figure 5(a) appear broader than the spectra in figure 5(b), even if the deposited films have the same thickness. This is due to the much thicker A20 substrate, whose effect on the $\alpha$-particle energy is much more pronounced. Sn samples usually show a more pronounced unevenness than Te ones, which can be intuitively observed by looking at the broader distribution of $\mathrm{Sn}$ data (orange dots in figure 6) with respect to Te ones (orange dots in figure 5).

The samples were also analyzed by Rutherford Backscattering Spectrometry at the AN2000 accelerator of the INFN Legnaro Laboratories. In the RBS measurements, a fraction of an accelerated alpha beam hitting the sample is partially backscattered at a certain angle. Such scattered beam is detected by a silicon detector that measures its energy. From the evaluation of the energy loss by the beam inside the target, the sample thickness can be deduced, together with the thickness of the buffer under the target layer, if present. Moreover, since the backscattered ions' energy strongly depends on the hit nucleus, RBS can be used to perform elemental analysis and to check the purity of the target. In the backscattering experiments the collection angle was $160^{\circ}$ and 


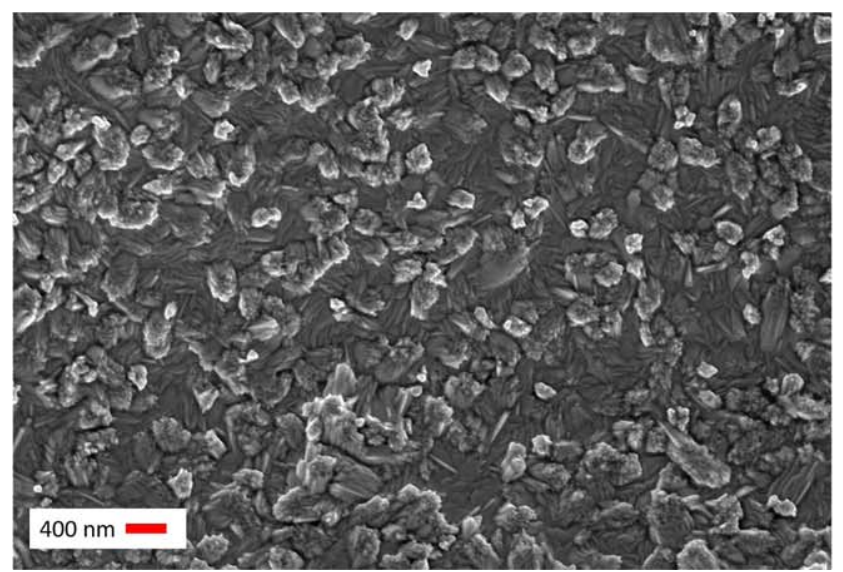

(a) SEM image of Te sample A14. Structure some hundred of nm top a uniform and compact background.

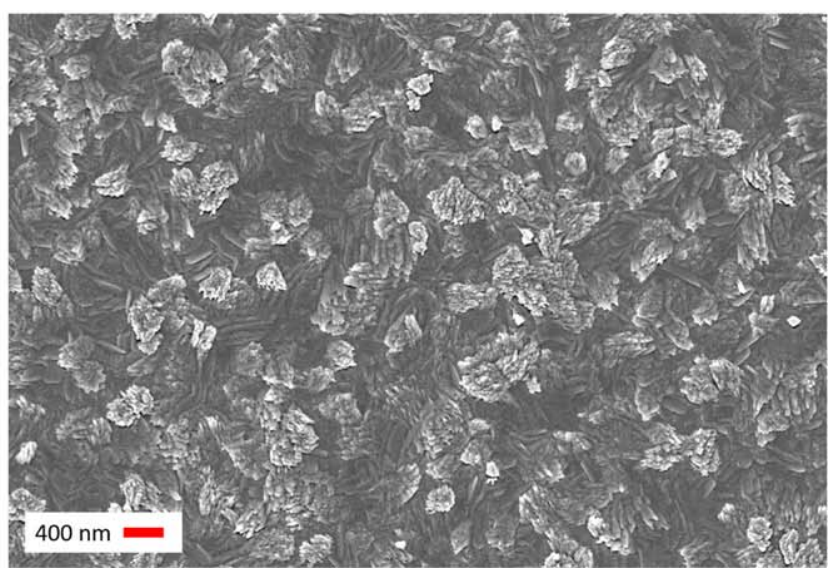

(b) SEM image of Te sample B10. The film appears to have a compact background, on which relatively small structures can be observed.

Figure 2. SEM images of Te samples A14 and B10. No relevant differences can be noticed.

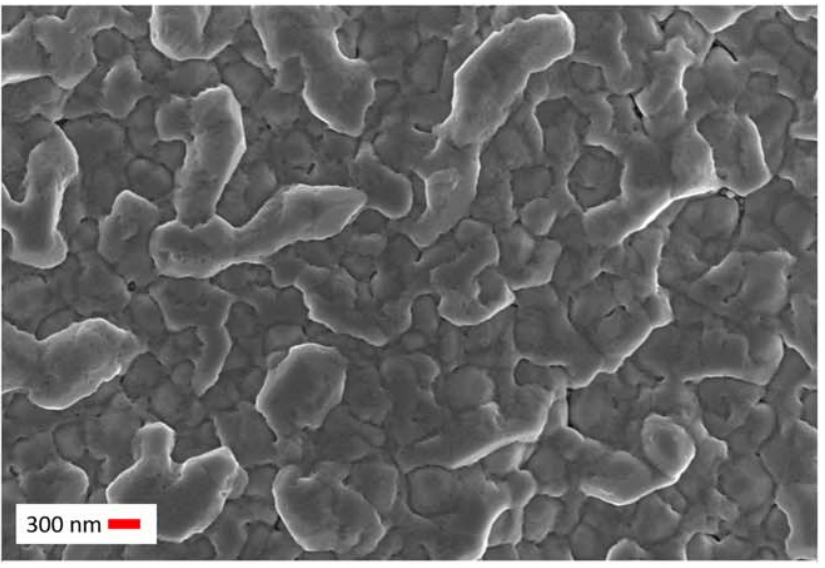

(a) SEM image of Sn sample A20. Large structures cover a compact background.

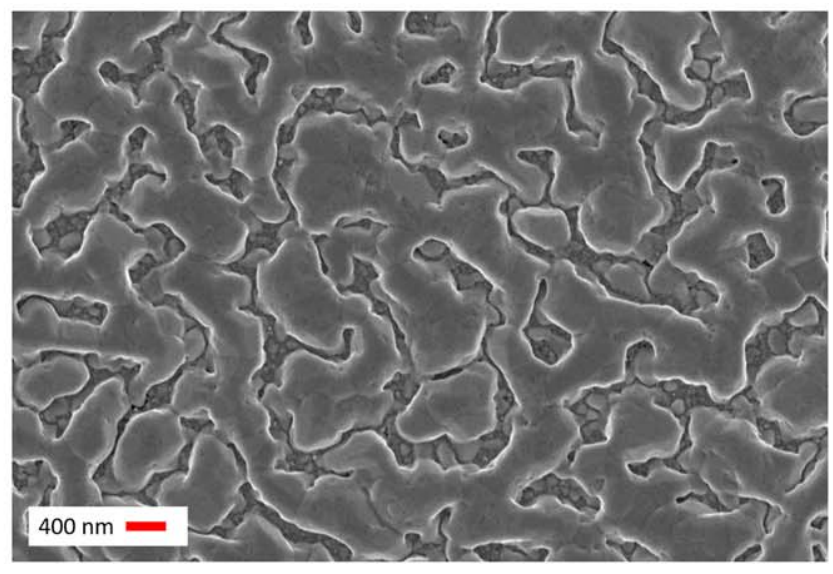

(b) SEM image of Sn sample B13. The overall flat film is run by deep trenches

Figure 3. SEM images of Sn samples A20 and B13. The two samples does not belong to the same production batch.

the $\alpha$-beam impinged on the samples at normal incidence with $\mathrm{E}_{\alpha}=2000 \mathrm{keV}$. As it can be seen in figures 7 and 8 , in the backscattering configuration the Te and Sn spectra can be analyzed individually (i.e. separated by HPOG substrate). Since the beam spot is of order of $1 \mathrm{~mm}^{2}$, the analyzed region is smaller than that of APT (where the analyzed area is about $7 \mathrm{~mm}^{2}$ ).

The thicknesses of the Te A14 and B10 samples were evaluated to be $268 \mu \mathrm{g} \mathrm{cm}^{-2}$ and $259 \mu \mathrm{g} \mathrm{cm}^{-2}$, respectively. The discrepancy between RBS and APT can be expressed as the difference of the measured values in relation to their average:

$$
\Delta=\frac{\left|x_{A P T}-x_{R B S}\right|}{\frac{x_{A P T}+x_{R B S}}{2}}
$$

Being $\mathrm{x}$ the measured thickness. The agreement with APT measurements is very good for sample A14 and good for sample B10 (around 3.4\%). The measured thickness for sample A20 was $182.5 \mu \mathrm{g} \mathrm{cm}^{-2}$, in good agreement with APT results (discrepancy of $12.8 \%$ ). Sample B13 resulted to be $116 \mu \mathrm{g} \mathrm{cm}^{-2}$, with a discrepancy of $11.5 \%$ with respect to APT value.

The analysis of the RBS spectra is complicated by the presence of a large roughness of both the substrate and the thin film. As pointed out in [6] and references therein, roughness produces measurable effects on the low energy edge of the elemental backscattering spectrum which is spread (see figure 8) because the surface and interface roughness results in variations of the layer thickness across the beam spot area. Moreover for targets with high roughness, the leading (high energy) edge of the spectrum may also be spread when the outgoing particle scattered from the rough surface may traverse asperities, exiting and re-entering the sample material more times. This second effect is mitigated at high backscattering angles and for normal incidence experiments like those used in this work. 


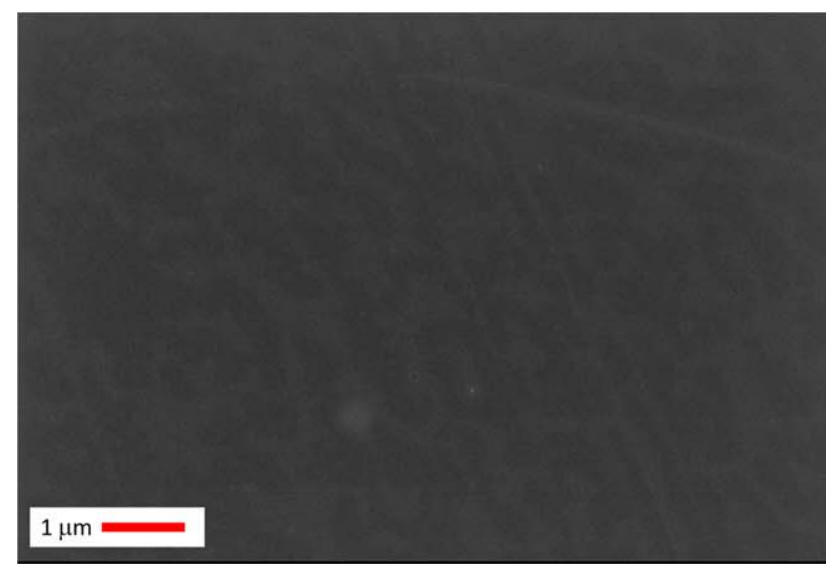

(a) SEM image of a HOPG sheet, $30 \mathrm{Kx}$ magnification.

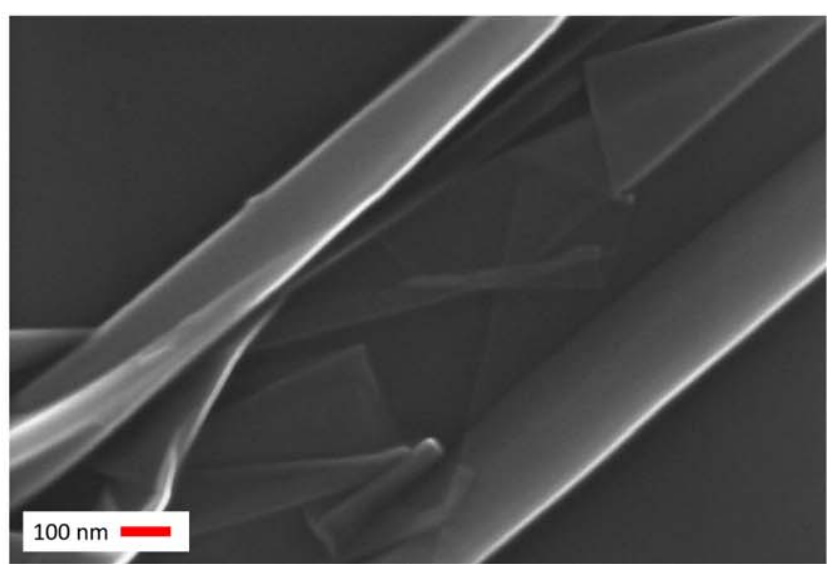

(b) Close up SEM image of a HOPG sheet, 150Kx magnification.

Figure 4. SEM image of a HOPG backing $1100 \mu \mathrm{g} \mathrm{cm}^{-2}$ thick. The sheet looks extremely flat, thanks to its crystalline structure (figure a). Difference in thickness may be due to oddly stacked graphite layers (figure b).

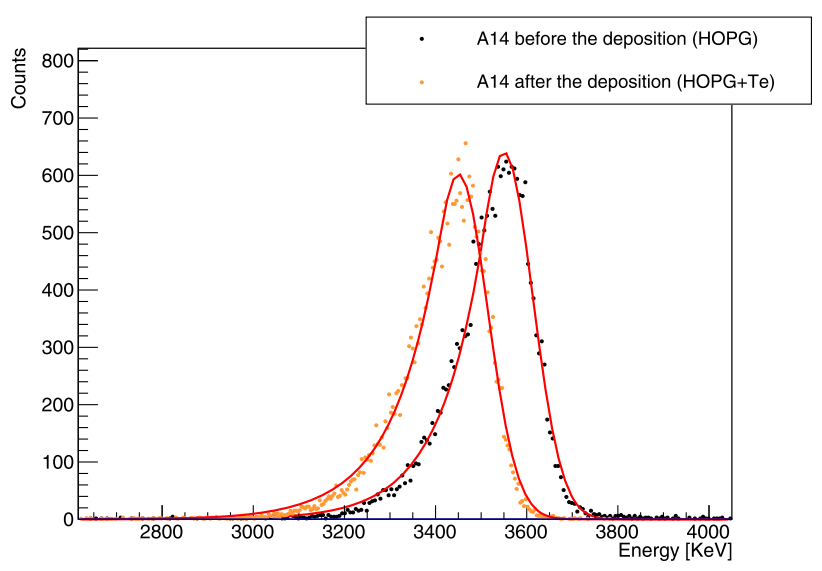

(a) APT measurement of sample A14, before and after deposition. Te film is $268 \mu \mathrm{g} / \mathrm{cm}^{2}$ thick, with a standard deviation of $16 \mu \mathrm{g} / \mathrm{cm}^{2}$. The points distribution can be fitted with a Crystal Ball function, pictured as red solid line.

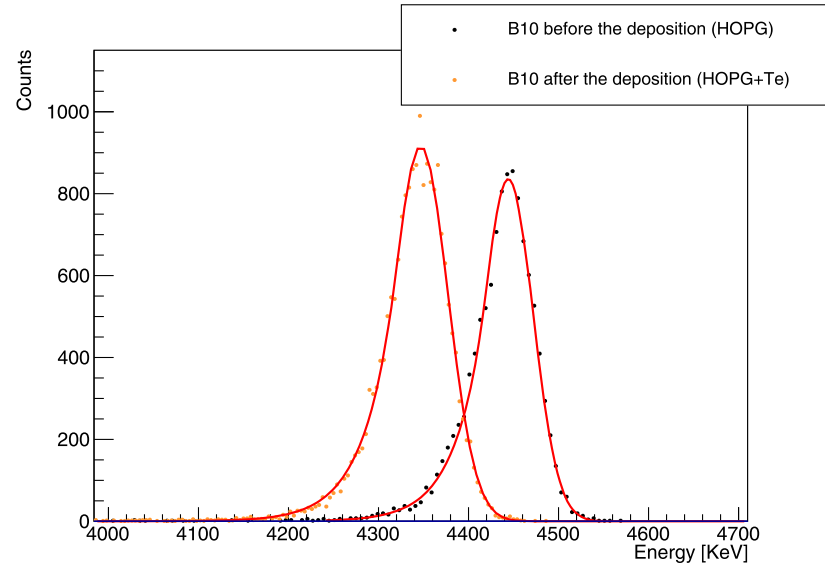

(b) APT measurement of sample B10, before and after deposition. Te film is $268 \mu \mathrm{g} / \mathrm{cm}^{2}$ thick, with a standard deviation of $29.5 \mu \mathrm{g} / \mathrm{cm}^{2}$. Data of the two sets are fitted with a Crystal Ball function, showed as a red solid line, properly fits the data of both sets.

Figure 5. APT analysis of samples A14 and B10, collected before (black dots) and after (orange dots) the deposition. Orange dots have a lower average energy due to the extra layer crossed.

As for the APT spectra, the RBS measurements confirm the fact that the Te targets are characterized by a better thickness uniformity than the Sn ones. The extraction of roughness distributions from combined APT and RBS measurements is a complex task and more work is ongoing to perfect the models used for fitting the APT and RBS data.

\section{Evaluation of energy resolution}

The energy of the projectile is affected by a number of effects, which are sources of uncertainty. Some are due to the involved physics, like straggling, while some other are due to the target morphology or to the equipment. The effects playing a major role in changing a particle energy are listed below.

- Superconducting Cyclotron: the energy of the ion beam is not perfectly monochromatic; particles' energy spread around the average value is about 1/1000th of the nominal energy. As a remark, the indicated value for the energy spreading is referred to the current setup; it will likely change after the programmed upgrade for the high beam intensity facility. A fine study on the magnetic optics and collimators is undergoing, in order to preserve (and hopefully decrease) the original energy spread. 


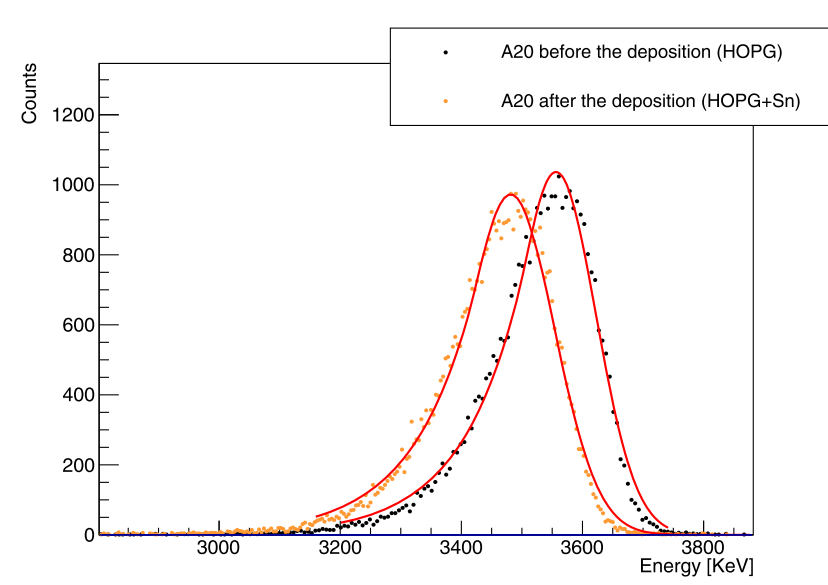

(a) APT measurement of sample A20, before and after deposition. Sn film is $160.6 \mu \mathrm{g} / \mathrm{cm}^{2}$ thick, with a standard deviation of $70.8 \mu \mathrm{g} / \mathrm{cm}^{2}$. Data can be fitted with a Crystal Ball function, pictured as red solid line.

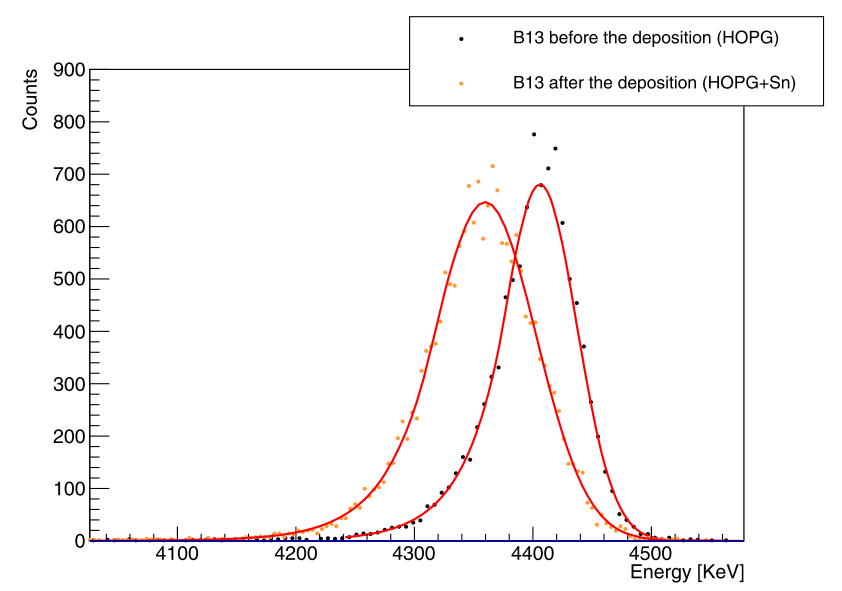

(b) APT measurement of sample B13, before and after deposition. Sn film is $131 \mu \mathrm{g} / \mathrm{cm}^{2}$ thick, with a standard deviation of $85.4 \mu \mathrm{g} / \mathrm{cm}^{2}$. Crystal Ball fits are well superimposed to data distributions.

Figure 6. APT analysis of samples A20 and B13, collected before (black dots) and after (orange dots) the deposition. The little shift of the orange dots toward lower energies is due to the limited thickness of the samples.

- MAGNEX Spectrometer: the error in the particles' energy measurement introduced by the dipole spectrometer, by the Si detectors and by the reconstruction algorithms is about $1 / 1000$ of the detected particle's energy. In the upgraded facility, the system shall be able to keep up with a much higher count rate, preserving the previous uncertainty on the energy measurements.

- Straggling: the straggling effect is due to a statistical variation of the number of small impacts with $\mathrm{e}^{-}$, suffered by unit path, by a charged particle crossing a medium. The outcome is a certain spread of the particles' energy around the average energy loss. In this particular context, things are complicated by the reactions under investigation. In a DCE event, the traveling charged particle suffers an increase (or decrease) of its atomic number $\mathrm{Z}$ by 2 units. Straggling, as well as the energy loss by ionization, depends on the projectile's Z, so that its magnitude differs sensibly before and after the DCE reaction. Moreover, straggling impact will strongly depend on where the reaction happens: an ${ }^{18} \mathrm{O}$ turning into a ${ }^{18} \mathrm{Ne}$ on the target surface will suffer more straggling with respect to an ${ }^{18} \mathrm{O}$ reacting at the target/ HOPG interface. In fact, it would cross a larger portion of target having a higher $\mathrm{Z}$. After the target, the ejectile has to cross the HOPG backing, whose nuclei $\left({ }^{n a t} \mathrm{C}\right)$ are much lighter than the target's. Straggling here will be once more different from earlier in the target.

- Dispersion: dispersion error arises from the impossibility of knowing the precise point in which the reaction will occur within the target. This is particularly important when studying DCE reactions, since the change in the projectile's Z number heavily influences the kinematics of its interaction with the target. The average energy loss differs substantially between a particle reacting at the target surface or at the target/HOPG interface. The first noticeable effect is a spectrum of average energy losses, one per infinitesimal portion of the target thickness. Every collection of particles reacting at the same depth, therefore having the same average energy loss, will suffer straggling as well as every other particle belonging to other average energy losses. The net effect is a further broadening of the energy distribution, due to the cross effect of dispersion and straggling.

- Thickness disuniformity: thickness disuniformity further broadens the shape of the energy distribution, worsening the effects of dispersion and straggling. Particles that cross a valley (i.e. impinges on a point of the target surface where the thickness is less than the target average thickness) will have a higher average energy that the rest of the particles. Conversely, particles crossing a bump (i.e. a spot thicker than the target average thickness) will lose more energy, spreading the energy distribution towards lower values. For this reason, the targets must be as flat as possible.

- Final state of reaction products: reaction products may exit the reaction in diverse excited states. Every state but the ground states will diminish the kinetic energy of the ejectile, effectively shifting the energy distribution toward lower energies.

Evaluating their cumulative effect on the ejectiles' energy distribution is somewhat tricky, since these effects cannot be evaluated independently. A statistical fluctuation on effect A (e.g. target thickness variation) can positively or negatively influence effect B (e.g. error due to unknown reaction depth). 


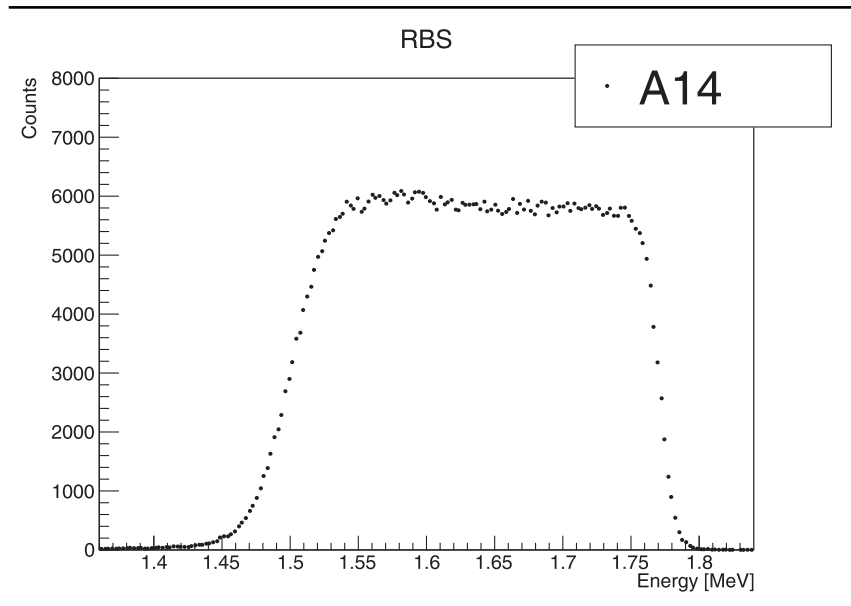

(a) RBS spectrum of sample A14, the thickness resulted to be $268 \mu \mathrm{g} / \mathrm{cm}^{2}$.

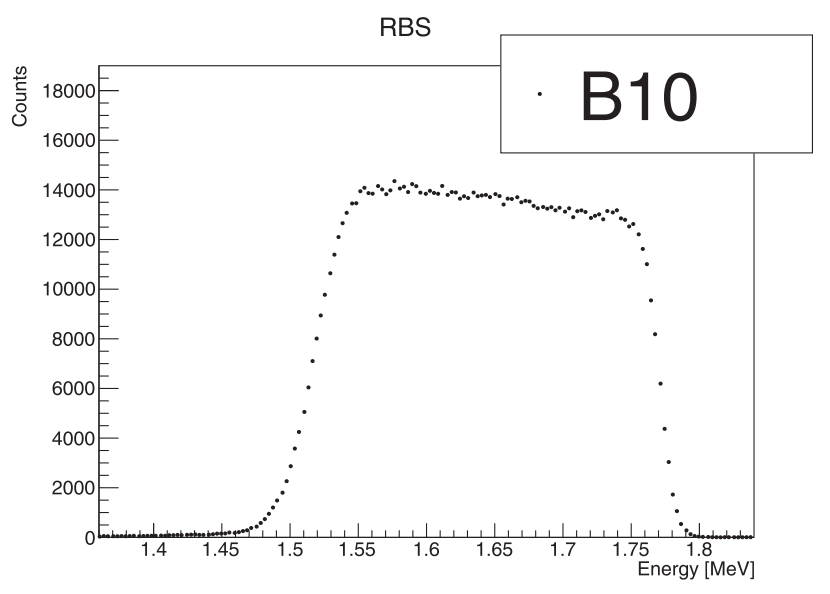

(b) RBS spectrum of sample B10, the thickness resulted to be $259 \mu \mathrm{g} / \mathrm{cm}^{2}$

Figure 7. RBS spectra of Te samples A14 and B10. In both the plots, the steepness of the slopes suggests a small unevenness of the depositions thickness.

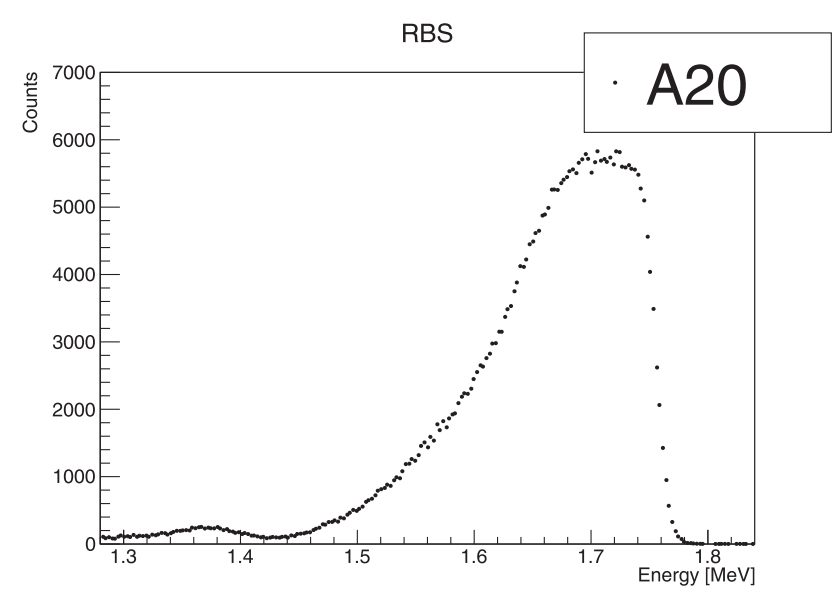

(a) RBS measurement of sample A20, the measured thickness being equal to $182.5 \mu \mathrm{g} / \mathrm{cm}^{2}$.

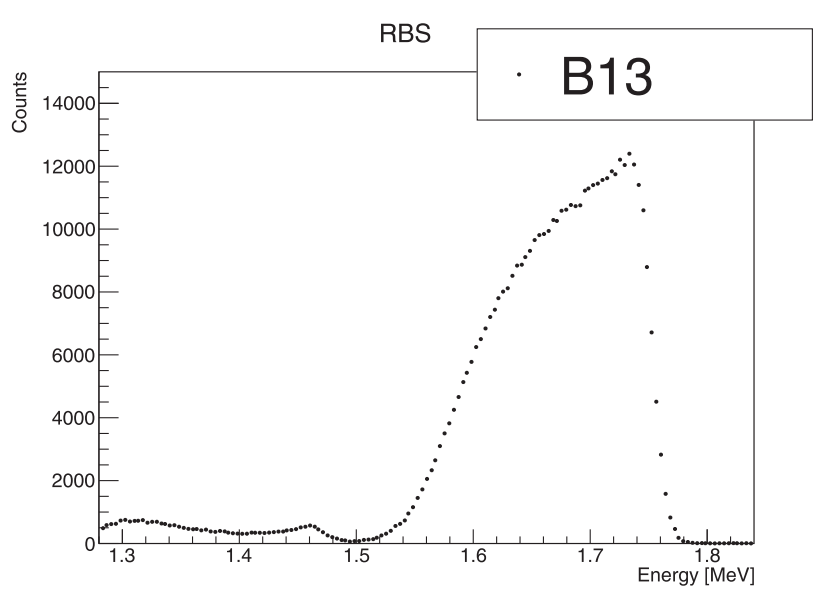

(b) B13 RBS spectrum, resulting to have a thickness of $116 \mu \mathrm{g} / \mathrm{cm}^{2}$.

Figure 8. RBS spectra of Sn samples B13 and A20. The relevant non uniformity detected by APT can be qualitatively observed in the long low energy tails of both the data sets. In both samples, a very thin $\mathrm{Cr}$ buffer is used to improve the adhesion between the film and the substrate; the $\mathrm{Cr}$ signal can be seen after the low energy tail of the $\mathrm{Sn}$ signal around $1.4 \mathrm{MeV}$, the position being influenced by the film thickness (not exclusively). In the B13 spectrum, a further bump can be seen at lower energies, due to impurities trapped during the deposition.

To evaluate the statistical and cumulative effects on reaction product energy, a Monte Carlo code was written.

The model has been designed to simulate step by step the energy loss of a particle within the target, randomizing the statistical events listed above using models or data from measurements when available.

\subsection{General remarks on Monte Carlo code}

The Monte Carlo code has been developed as a standalone program, but it will be integrated into NUMEN simulation software package to improve its accuracy. Some changes are in order to match the experimental needs and to fit the already existing software, but the code will be mostly preserved. The necessary input parameters are listed below.

- The target isotope is used by designated functions to automatically load a set of quantities related to the chosen reaction channel, $\left({ }^{18} \mathrm{O},{ }^{18} \mathrm{Ne}\right)$ or $\left({ }^{20} \mathrm{Ne},{ }^{20} \mathrm{O}\right)$. Depending on the latter, the beam species and the reaction products are set up, together with their characteristic quantities (atomic number, mass, energy levels, density). An optional sample label can be added to improve readability;

- The target and substrate thickness and roughness, either coming from APT measurements or guessed to estimate the resolution of a foreseen target; 
- Projectile energy, expressed in $\mathrm{MeV} / \mathrm{u}$;

- Number of DCE events, set by default to $2 \cdot 10^{6}$.

The energy loss of each simulated particle is evaluated stepwise by using the Bethe-Bloch formula (equation (6)), keeping fixed the infinitesimal energy loss $\delta E$ (default values set equal to $5 \cdot 10^{-5} \mathrm{MeV}$ ); the spacial increment $d x$ varies accordingly. The quantities inside the formula are adjusted depending on the particle's position (the crossed medium quantities varies between target and substrate) and history (e.g. its $\mathrm{Z}$ depends on whether the DCE reaction already occurred).

Given the energy involved, high energy corrections are neglected and the formula reads:

$$
-\frac{d E}{d x}=\frac{4 \pi N_{A} r_{e}^{2} m_{e} c^{2} \rho}{\beta^{2}} z^{2} \frac{Z}{A}\left(0.5 \ln \frac{\beta^{2} \gamma^{2} E_{\max }}{I^{2}}-\beta^{2}\right)
$$

where $N_{A}$ the Avogadro's Number, $m_{e}$ and $r_{e}$ the electron's mass and radius; $\rho, I, A$ and $Z$ are the medium's density, mean excitation potential, atomic number and mass number, respectively; $z$ the projectile atomic number. Finally, $E_{\max }$ is the maximum energy transferable to an electron in a single collision, defined as:

$$
E_{\max }=\frac{2 m_{e} \beta^{2} \gamma^{2}}{1+2 \gamma \frac{m_{e}}{m_{x}}+\frac{m_{e} 2}{m_{x}}}
$$

being $m_{x}$ the projectile mass.

\subsection{Monte Carlo code structure}

A short scheme of the present version of the code is provided here.

\section{- Target and beam parameters}

Thickness and relative unevenness from APT measurements (see section 3) are used to guess the path crossed by the simulated ion. The effective thickness is sorted from the Gaussian distribution built with the measured average thickness and standard deviation of the sample. The same procedure is applied to sort the effective HOPG thickness. The reaction depth at which the DCE reaction occurs is sorted within the previously determined effective target thickness. Due to the very small thickness, the reaction probability is assumed constant throughout the target: the actual value of cross section is unknown and is the goal of the NUMEN experiment.

The particle's energy is also modified due to the SC beam energy spread. The energy distribution of the SC beam can be assumed to follow a Gaussian, whose standard deviation $\sigma_{S C}$ is related to the FWHM of the beam spot by: $F W H M=2 \sqrt{2 \ln 2} \sigma_{S C}$ ). The FWHM of the $\mathrm{SC}$ beam at various mean energies $E_{b}$ is proportional to $E_{b}$ by a factor $10^{-3}$. Therefore, the value used for $\sigma_{S C}$ in the MC is: $\sigma_{S C}=E_{b} \cdot 10^{-3} / 2 \sqrt{2 \ln 2}$.

- Evaluation of straggling before DCE

The energy loss suffered by the projectile is calculated by using the Bethe-Bloch formula (equation (6)). Fixed very small energy steps, corresponding to very small spatial increments, are repeatedly added until the particle reaches the randomly sorted reaction depth, still having a certain residual energy. Before the reaction, straggling is evaluated using the Gaussian model [7]:

$$
\sigma_{\text {straggling }}=\sqrt{\xi E_{\max }\left(1-\beta^{2} / 2\right)}
$$

where $E_{\max }$ was defined above (paragraph 4.1) and $\xi$ is the mean energy loss, defined as:

$$
\xi=\frac{2 \pi e^{4} N_{A} z^{2} Z \rho \delta x}{m_{e} \beta^{2} c^{2} A}
$$

Where $e$ is the electron charge and the other quantities were defined in paragraph 4.1 .

The energy of the particle is updated by sorting a value in a Gaussian distribution centered around the residual energy and having as standard deviation $\sigma_{\text {straggling. }}$

\section{- DCE reaction}

The energy of the ejectile exiting the DCE reaction is obtained by computing the kinematics of the collision $X_{A}^{B}\left(y_{a}^{b}, y_{a}^{*}{ }_{a} \pm 2\right) X_{A}^{* B \mp 2}$, supposing that both the recoiling nucleus and the ejectile can exit the reaction in an excited state. The first three energy levels are considered for each involved nucleus. Considering a flat equal probability, there is a total of 9 alike combinations. So far, only reactions in which the projectile exits at 0 degrees have been simulated.

\section{- Evaluation of straggling after DCE}

After the reaction, ejectile's straggling and energy loss are calculated for the residual depth of the target and for the HOPG backing. The procedure followed is the same used before the reaction, considering atomic number change of the traveling particle due to the DCE. Straggling is sorted twice here, once after the target and one after the HOPG substrate.

\section{- MAGNEX Resolution}

The last contribution to be added is due to the MAGNEX spectrometer and particles' trajectory reconstruction procedure. Similarly to the Cyclotron, MAGNEX error can be quantified by using the FWHM value $F W H M=$ $E_{\text {ion }} \cdot 10^{-3}$, here considering the ion's energy after it's passed the target assembly. The energy of the ion is once again modified guessing a value within a Gaussian distribution, centered around the ion's energy and having $\sigma_{M A G N E X}$ as standard deviation (being $\sigma_{M A G N E X}$ defined similarly to the SC case: $\sigma_{M A G N E X}=E_{\text {ion }} \cdot 10^{-3} / 2 \sqrt{2 \ln 2}$ ).

\section{- Sorting of simulated particles}

The energies of the simulated particles are collected into 10 vectors, one per each combination of excited levels plus one containing the energy of every simulated particle. This sorting proves useful when calculating the standard deviation of each energy distribution and the related FWHM, a necessary step in evaluating the global energy resolution. In fact, each the standard deviation is used to build a Gaussian, which is superimposed to each distribution. Using the Gaussian fits, the FWHM of each 


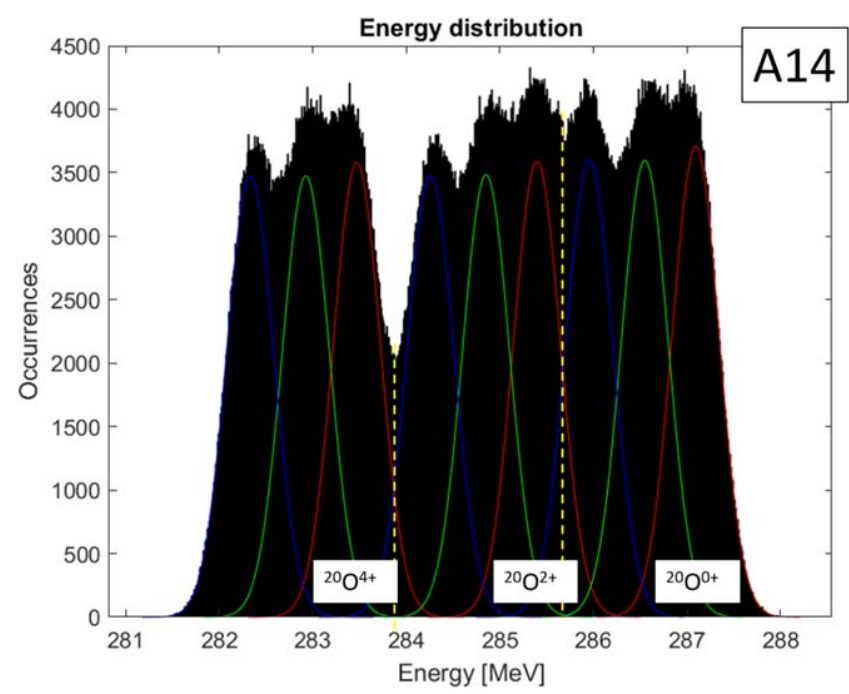

Figure 9. Energy distribution outcome of Monte Carlo simulation of sample A14. The G.S. and the first two excited levels of ${ }^{130} \mathrm{Xe}$ are fitted, respectively, with red, green and blue coloured Gaussians. The red curves are centered around the energy shift due to the final state of the ejectile ${ }^{20} \mathrm{O}$, the green and blue ones follow at lower energies. The triplets of the target's states are separated by yellow dotted lines.

distribution is calculated and then used to evaluate the energy resolution. To distinguish two adjacent energy levels, it is necessary for the resolution to be smaller than their energy gap. For two adjacent energy distribution, this criteria translates into:

$$
\Delta E_{\text {res } 1,2}=\frac{F W H M_{1}+F W H M_{2}}{2}<E_{\text {gap } 1,2}
$$

Where $\Delta E_{\text {res } 1,2}$ is the resolution between level 1 and 2 , FWHM $_{i}(\mathrm{i}=1,2)$ the FWHM of the ith distribution and $E_{\text {gap } 1,2}$ is the energy gap between levels 1 and 2 .

\subsection{Results from simulations}

Using the results obtained from measurements of the described prototypes, the final energy resolution was simulated. For the simulations, the characteristics of the isotope ${ }^{130} \mathrm{Te}$ and ${ }^{130} \mathrm{Xe}$ (the recoiling nucleus of the DCE) were considered. In the showed plots, the lowest energy states combination (i.e. ejectile and recoiling nucleus in Ground State) is the rightmost peak; higher energy states combinations appear at lower energies. The total energy distributions of Te samples A14, figure 9, and B10, figure 10, are shown.

Ground states, first excited and second excited of the recoiling nucleus are fitted with red, green and blue coloured Gaussian, respectively. They are energy-shifted depending on the ejectile energy level. The deposited films of Te are very similar, the only two differences lying in the disuniformity (6\% for A14 and $11 \%$ for B10) and the thickness of the backing (2200 $\mu \mathrm{g} \mathrm{cm}^{-2}$ and $1100 \mu \mathrm{g} \mathrm{cm}^{-2} \mathrm{HOPG}$, nominal thickness). The contribution of the backing thickness is apparent: despite B10 higher roughness, by halving said thickness the resolution $\Delta E_{\text {res } 0,1}$ for G.S. and first excited level of ${ }^{130} \mathrm{Te}$ (the ejectile ${ }^{20} \mathrm{O}$ in G.S.) decreases by nearly

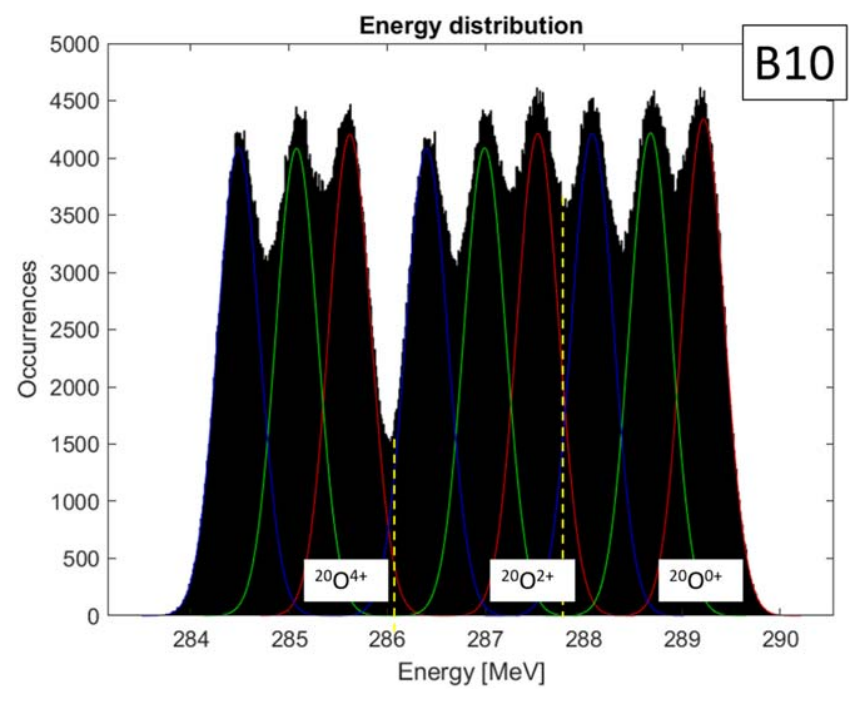

Figure 10. Energy distribution outcome of Monte Carlo simulation of sample B10. The colour scheme used for sample A14 is used here as well. Due to the thinner backing, the peaks seem more distinguishable with respect to figure 9 .

$70 \mathrm{keV}$. More specifically, $\Delta E_{\text {res } 0,1}=654.2 \mathrm{keV}$ for $\mathrm{A} 14$ and $\Delta E_{\text {res } 0,1}=579 \mathrm{keV}$ for $\mathrm{B} 10$. The achieved resolution is not sufficient, yet. The energy gap $E_{\text {gap } 0,1}$ of ${ }^{130} \mathrm{Xe}$ is in fact $536.1 \mathrm{keV}$; a further reduction of the target and backing thickness is needed.

For what it concerns $\mathrm{Sn}$ prototypes, the comparison between sample B13 with sample B6 (described in [4]) can be instructive about the role played by non uniformity. Achieving a sufficient resolution for Sn target is particularly problematic, mainly for two reasons: the ${ }^{116} \mathrm{Cd}$ energy gap narrowness $\left(\Delta E_{\text {res } 0,1}=513.5 \mathrm{keV}\right)$ and the growing mechanism of Sn during the deposition, which favors large material clusters rather than a uniform material distribution. In figure 11 are shown the first three energy levels of ${ }^{116} \mathrm{Cd}$ paired with the ejectile in G.S. for prototypes B6 (figure 11(a)) and B13 (figure 11(b)).

The two samples have a very different morphology: B6 is $234.3 \mu \mathrm{g} \mathrm{cm}^{-2}$ thick with a disuniformity of $65.6 \mu \mathrm{g} \mathrm{cm}^{-2}$, while $\mathrm{B} 13$ is $131 \mu \mathrm{g} \mathrm{cm}^{-2}$ with an unevenness of $85.4 \mu \mathrm{g} \mathrm{cm}^{-2}$. Despite B13 being much thinner than B6, its energy resolution is heavily affected by the higher degree of non uniformity. For B13, resolution between ${ }^{116} \mathrm{Cd}$ G.S. and its closest energy state is $\Delta E_{\text {res } 0,1}=625.8 \mathrm{keV}$, while the same quantity for sample B6 is equal to $\Delta E_{r e s} 0,1=588.8 \mathrm{keV}$.

From the simulation results showed in figure 11 , it is apparent the importance of reducing the unevenness as much as possible, in order to maximize the deposition thickness and, therefore, the reaction rate.

\section{Procedure for samples' design}

Combining all the steps exposed so far it is possible to establish a standard procedure to produce targets which meet the experimental requirements. 


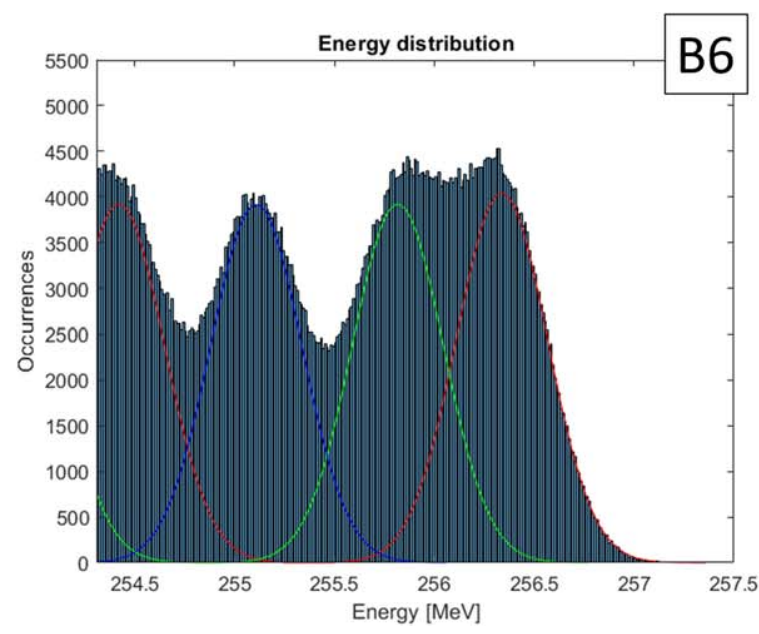

(a) Energy distribution of ejectiles ${ }^{18} \mathrm{Ne}$ leaving ${ }^{116} \mathrm{Cd}$ recoiling nuclei in G.S. (red line), first excited level (green line) and second excited level (blue line) in sample B6.

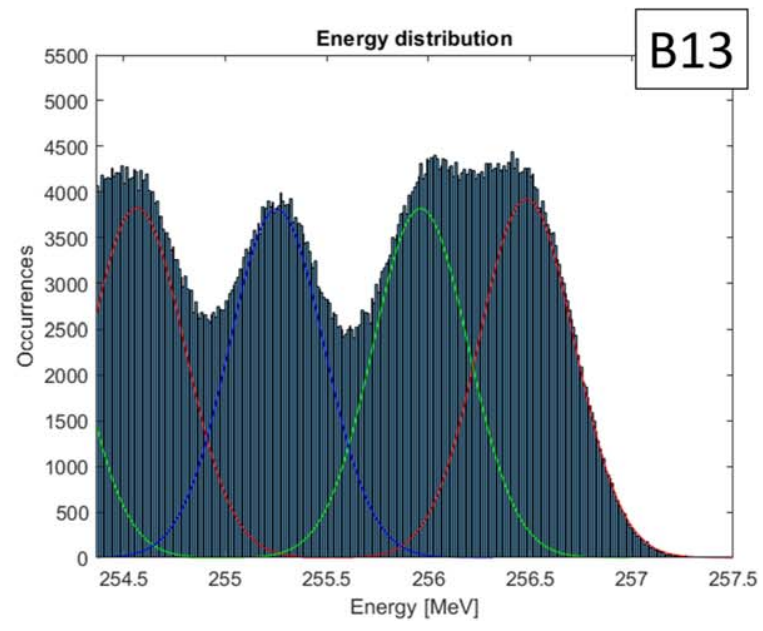

(b) Energy distribution of ejectiles ${ }^{18} \mathrm{Ne}$ leaving ${ }^{116} \mathrm{Cd}$ recoiling nuclei in G.S. (red line), first excited level (green line) and second excited level (blue line) in sample B13.

Figure 11. A comparison between energy distribution of ejectiles exiting samples B6 and B13. The distributions look similar, despite the differences in the samples' morphology.

- Substrates characterization: HOPG substrates, purchased by an external company, are characterized by using APT, in order to assess their effective thickness and disuniformity.

- Exploratory deposition: The element under study is deposited on HOPG substrates $2200 \mu \mathrm{g} \mathrm{cm}^{-2}$ and $1100 \mu \mathrm{g} \mathrm{cm}^{-2}$ thick (nominal values). Thinner substrates, about $450 \mu \mathrm{g} \mathrm{cm}^{-2}$ are currently under study.

- Sample characterization: prototypes are characterized by using the techniques described in section 3 .

- Energy resolution simulation: results of characterization are used to simulate the final energy resolution. If the resolution must be improved, new deposition conditions are explored until satisfactory results are achieved.

The combination of suitable characterization techniques and simulation code is valuable in pointing out which specific target feature must be changed in order to improve the energy resolution. The code can be used to see the outcome after changing one or more parameters of the target or of the substrate, providing a lead on where to focus the efforts to obtain the desired resolution.

\section{Conclusions and future research}

The need for statistically valid data in the measurement of DCE cross-section in the NUMEN experiment calls for the use of a high intensity ion beam. The targets need to be backed by a highly thermally conductive HOPG substrate, which negatively affects the energy resolution of the collected particles. Since preserving a sufficient energy resolution is as important as the high statistics, a careful study was performed on target prototypes to reach the required precision. Te and Sn prototype were deposited by Electron Beam Evaporation on HOPG substrate of different thickness and then thoroughly characterized with Scanning Electron Microscopy, AlphaParticle Transmission, and Rutherford Backscattering Spectrometry techniques. Once the targets' thickness and unevenness were assessed, they were used as input parameters of a Monte Carlo code, written to account for the major effects which affect the particles' energy. Thanks to the synergy of experimental characterization techniques and simulations, it is possible to predict the effect of the target on the total energy resolution. The program can be used to see what physical characteristic of the target is better to adjust to obtain the maximum improvement. A procedure to produce targets suitable for the experimental requirements has been established and described. Future experimental work include a deeper study on the thinnest available HOPG substrate ( $450 \mu \mathrm{g} \mathrm{cm}^{-2}$ ), the improvement of Te and Sn non uniformity and the study of other target materials. The code will be tested using data collected during past NUMEN runs and recently analyzed. Real data will prove of great help in improving the code accuracy.

\section{References}

[1] Cavallaro $\mathrm{M}$ et al 2020 The MAGNEX magnetic spectrometer for double charge exchange reactions Nucl. Inst. and Methods B 463 334-8

[2] Cappuzzello F et al 2018 The NUMEN project: NUclear Matrix Elements for Neutrinoless double beta decay Eur. Phys. J. A 5472

[3] Iazzi F et al 2017 A new cooling technique for targets operating under very intense beams WIT Trans. Eng. Sci. 116 61-70 
[4] Capirossi V et al 2020 Thickness and Uniformity

Characterization of Thin Targets for Intense Ion Beam Experiments Acta Phys. Pol. B 51661

[5] Gaiser J E 1982 Appendix-F Charmonium Spectroscopy from Radiative Decays of the J/Psi and Psi-Prime Ph.D. Thesis Stanford University (https://www-public.slac.stanford.edu/ sciDoc/docMeta.aspx?slacPubNumber=slac-R-255)
[6] Molodtsov S L et al 2008 Accurate ion beam analysis in the presence of surface roughness JoPD 4120

[7] Seltzer S M and Berger M J 1964 Energy loss straggling of protons and mesons Studies in Penetration of Charged Particles in Matter (Nuclear Science Series 39) (Washington DC: Nat. Academy of Sciences) (https://doi.org/10.17226/ 20066) 